УДК $378+51-7+330.4$

\title{
ЭНТРОПИЙНЫЙ ПОДХОД В ОЦЕНКЕ ЭФФЕКТИВНОСТИ ИННОВАЦИЙ В ОБРАЗОВАТЕЛЬНОЙ СРЕДЕ
}

\author{
Бояров Е.Н., Абрамова С.В.
}

В статье рассматривается актуальная проблема квалиметрии инноваций в образовательной среде. Поскольку в теории информации для определения степени неполноты, неопределённости знаний в информационной системе рассчитывают ее энтропию, в качестве меры показателя образовательной системы предлагается использовать уровень энтропии в баллах, что сделает процесс и анализа более простым, а его результат более доступным для практического применения в конкретной образовательной среде. Критерием для оценки уровня энтропии в системе высшего профессионального образования может служить падение уровня сформированности предметных понятий. При этом критическому уровню энтропии будет соответствовать практически полное отсутствие сформированности предметных понятий. Таким образом, предлагается на основе энтропийного подхода оценить ожидаемый дидактический эффект от внедрения различных инновационных образовательных технологий в систему высшего профессионального образования.

Ключевые слова: энтропийный подход, образование, информация, социальная информация, образовательная технология.

\author{
THE INNOVATION OF THE EDUCATIONAL ENVIRONMENT \\ ASSESSMENT EFFICIENCY BASED OF THE ENTROPY APPROACH
}

Boyarov E.N., Abramova S.V. 
The article is revealed actual problems of qualimetry of innovations in the educational environment. In information theory to determine the degree of incompleteness, uncertainty of knowledge in the information system calculated its entropy. As a measure of the indicator of the educational system is proposed to use the level of entropy in marks. This will make the process easier and analysis, and its results more accessible for practical use in a particular educational environment. The criteria for assessing the level of entropy in the system of higher education can serve as a drop in the level of formation of subject concepts. In this critical level of entropy will correspond to the almost complete absence of formation of subject concepts. So it's proposed to estimate the expected effect of a didactic introduction of various innovative technologies in the educational system of higher education based on the entropy approach.

Keywords: entropy approach, education, social information, educational technology.

Термодинамика XX века изучает открытые системы в состояниях, далеких от равновесия. Это направление получило название синергетика (от «синергия» - сотрудничество, совместное действие).

Самоорганизация, положенная в основу синергетики, по определению ее автора, немецкого физика Германа Хакена, - есть «спонтанное образование высокоупорядоченных структур из зародышей или даже из хаоса, спонтанный переход от неупорядоченного состояния к упорядоченному за счет совместного, кооперативного (синхронного) действия многих подсистем» [7].

Развитие понимается в синергетике как процесс становления качественно нового, того, что еще не существовало в природе и предсказать которое невозможно. Для отражения этого процесса в термодинамику было введено новое понятие - энтропия. Под энтропией стали понимать меру беспорядка системы. Физический смысл возрастания энтропии сводится к тому, что состоящая из некоторого множества частиц изолированная (с постоянной энергией) система 
стремится перейти в состояние с наименьшей упорядоченностью движения частиц. Это - наиболее простое состояние системы, или состояние термодинамического равновесия, при котором движение частиц хаотично. Максимальная энтропия означает полное термодинамическое равновесие, что эквивалентно полному хаосу [5].

Статистическая физика рассматривает как меру вероятности пребывания системы в данном состоянии, то есть, как функцию состояния, когда любому состоянию можно сопоставить вполне определенное значение энтропии.

В информатике энтропия определяется как степень неполноты, неопределённости знаний. Основоположник теории информации К. Шеннон и основоположник кибернетики Н. Винер отмечали тесную связь информации и энтропии.

Если К. Шеннон (1948 г.) под информацией понимает сообщение, уменьшающее неопределенность (энтропию) у получателя сообщений, то Н. Винер, в этом же году пишет, что «количество информации, будучи отрицательным логарифмом величины, которую можно рассматривать как вероятность, по существу есть некоторая отрицательная энтропия» [3].

Таким образом, они сформулировали энтропию как меру хаоса в противовес количеству информации являющейся мерой, определяющей порядок системы.

Качественно, чем выше энтропия, тем в большем числе существенно различных микросостояний может находиться объект при данном макросостоянии. При вычислении энтропии требуется математическая модель объекта и его фазового пространства. Математическая модель содержит атрибуты двух типов. Атрибуты, инвариантные относительно всех допустимых преобразований модели, называются структурными. Они образуют структуру модели. К ним относятся уравнения, описывающие объект, пространство, из которого берут свои значения переменные, алгебра и топология на этом пространстве, система начальных, граничных условий и т. п. 
Другие атрибуты математической модели могут изменять свои значения при разных преобразованиях. Это вариативные параметры. К ним относятся значения переменных, координатные системы, в которых записаны уравнения, изменяемые связи между частями модели, границы областей, где ищутся решения и т. п. Полная совокупность всех вариативных параметров называется фазовым состоянием модели (его общей записью), а набор конкретных значений этих параметров - фазовой точкой или микросостоянием. Совокупность всех возможных фазовых точек называется фазовым пространством модели. Обычно, на таком пространстве можно ввести естественную метрику или топологию.

Принято считать, что любая система стремится к состоянию равновесия, т. е. растет энтропия системы. Однако второе начало термодинамики (закон сохранения энтропии) требует компенсировать рост энтропии. Информация и является средством компенсации. Большинство систем открыты (И. Пригожин, И. Стенгерс) - они обмениваются энергией, вещество и информацией с окружающей средой.

Энтропия дает числовую меру неопределенности. Поэтому в любом определении энтропии присутствует математическое описание объекта и его состояния, а также дополнительные характеристики модели, позволяющие измерить неопределенность.

Рассматривая систему высшего профессионального образования во взаимосвязи с образовательной средой, с точки зрения социокоммуникативного подхода (Д.Ю. Астапенко, 2010; Т.А. Магеррамова, 2008) необходимо выделить в её составе информационную сущность, роль которой будет играть социальная информация.

Социальная информация - совокупность знаний, сведений, данных и сообщений, которые формируются и воспроизводятся в обществе и используются индивидами, группами, организациями, различными социальными институтами для регулирования социального взаимодействия, общественных отношений и процессов. 
Социальная информация низшего уровня представляет собой модели хотения, опасения, намерения, информационного и реального действия одного человека по отношению к другому человеку. То, что хочет один человек передать другому человеку, каким образом это передать и исполнение намерений информационным и реальным действием - это только одна сторона обменных процессов, происходящих между двумя людьми. Другая сторона обменных процессов будет представлять собой информацию о том, что ожидает этот человек от другого человека в качестве его хотения, его опасения, его намерения, его информационного и реального действия. Такой обмен социальной информации, есть управление человеком человека (в явной или неявной формах).

Социальная информация передаётся с помощью различных информационных средств, которые принято называть языком - понятийным аппаратом. В условиях современного общества как социальной системы представляется возможным говорить о социальной энтропия, представляющей собой количественный фактор нестабильности общества (или, кок её элемент - отдельной социальной группы).

При этом под социальной энтропией системы образования будем понимать меру отклонения социальной системы (например, системы высшего профессионального образования) или ее отдельного звена (системы предметной подготовки, системы усвоения предметных понятий) от принятого как эталонное (нормальное, ожидаемое) состояния (максимальная образованность, полное усвоение учебного материала), которое (отклонение) проявляется в снижении уровня организации, эффективности функционирования, темпов развития этой системы. Социальная энтропия связана с наличием объективной неопределенности в состоянии среды, образования людей, недостатком знаний (информации) в процессе организации рассматриваемой системы - общества в целом или образовательной среды, в частности.

Энтропия знаний - в смысле мера отсутствия уверенного (на данном этапе развития личности) знания о смысле известных по названию понятий, фак- 
тов реального положения дел в окружающей действительности, взаимосвязях фактами и фактами - понятиями и фактами, понятиями и понятиями. В силу конечности и ограниченности человеческого знания, энтропия знания никогда не равняется нулю.

Для задачи высшего профессионального образования трактовку энтропии можно провести через вероятность нахождения обучаемого на некотором фиксированном уровне фазового пространства обучения внутри системы. Отсюда видно, что понятие энтропии играет существенную роль как своеобразная мера упорядоченности внутри системы обучения [6].

Таким образом, уровень энтропии позволяет охарактеризовать степень организованности знания в предметной области, т.е. дает возможность оценить эффективность образовательной системы в образовательной среде на основе энтропийного подхода. Суть подхода состоит в том, что любые системы обладают одним общим показателем - уровнем энтропии, который отражает состояние системы и динамику ее развития. Соответственно, изменение этого уровня в результате внедрения инноваций (образовательных инноваций - качественно нового) может служить критерием для их оценки [4].

В теории (гипотетически) применение инновации в работе системы должно снижать уровень ее энтропии и не допустить возрастание ее до некоторого критического уровня, при котором состояние системы будет характеризоваться как хаотичное (в примере образовательной системы - будет соответствовать отсутствию предметных знаний, хаотичности, т.е. отсутствием системности знаний). При этом возрастание энтропии системы происходит как под влиянием внутренних противоречий (в субъекте образовательной среды, структура и содержание образовательной технологии в образовательной среде и др.), так и под влиянием внешних факторов (социально-экономические условия, запросы общества, социальный заказ и др.).

При этом текущая образовательная деятельность в среде позволяет лишь поддерживать уровень энтропии на приемлемом уровне в течение определенно- 
го времени. Поскольку в соответствии с законом о неубывании энтропии в замкнутой системе происходит монотонное возрастание энтропии, в любом случае может наступить момент, когда потребуются кардинальные меры по её снижению. Так, на примере образовательной системы (субъект образования предметные знания), потребуются образовательные инновации, причем как технологические, так и управленческие, направленные на интенсификацию данного процесса (то есть, направленные на снижение энтропии). Примером применения образовательных инноваций может служить применение в образовательной среде информационно-коммуникационных технологий, технологий дистанционной поддержки образовательного процесса, технологий виртуальной вузовской среды и др.

Критерием для оценки уровня энтропии в системе высшего профессионального образования может служить падение уровня сформированности предметных понятий. При этом критическому уровню энтропии будет соответствовать практически полное отсутствие сформированности предметных понятий.

Для оценки эффективности инноваций важно оценивать именно изменение уровня энтропии в затрагиваемых системах, а не текущий ее уровень, что позволяет значительно облегчить расчеты. При этом учитывается, что энтропия при переходе из одного состояния в другое не зависит от пути перехода. Иначе говоря, следует определить не уровень энтропии, а степень ее изменения.

В естественных науках разработаны методы измерения энтропии, однако для целей педагогического анализа можно применять более простые методы например, оценивать уровень энтропии в баллах, что сделает процесс и анализа более простым, а его результат более доступным для практического применения в конкретной образовательной среде. При применении метода балльной оценки энтропии сначала задается граница возможных изменений энтропии. Например, текущему уровню энтропии можно приписать значение 0 баллов а критическому (максимально допустимому) уровню энтропии - значение 100 
баллов. Предполагается, что превышение этого уровня приведет к разрушению анализируемой системы.

Затем выбирается ключевой показатель (группу показателей), наиболее полно характеризующий уровень энтропии в системе, текущее (либо среднее) его значение приравнивается нулю, а предельно допустимое - к 100. Соответственно, у каждого изменения показателя (взятого по модулю) появляется цена (энтропийный эффект), выраженная в баллах. Например, для образовательной системы в качестве ключевого показателя можно принять уровень сформированности предметных знаний. Допустим, текущий уровень сформированности составляет $30 \%$, критический - 5\%. Значит, цена одной единицы показателя сформированности предметных знаний в процентах составит $100 /(30-5)=4$ балла. Отсюда, если прирост предметных знаний в результате внедрения инновации составит 10\%, это даст снижение энтропии на 40 баллов [4].

У различных энтропийные эффектов разный масштаб и разная вероятность осуществления. Масштаб энтропийного эффекта зависит от категории инноваций и масштаба оценки. Для удобства расчетов следует ограничиваться, например, рамками одной образовательной среды. Тогда масштаб инноваций будет выражаться в доле затрагиваемых данной инновацией соответствующих подсистем в рамках конкретной образовательной среды.

Таким образом, масштаб эффективности применения инновации в образовательной среде будет выражаться как доля обучающихся, имеющих заданный уровень сформированности предметных знаний.

Вероятность осуществления энтропийных эффектов зависит от горизонта предвидения и степени неопределенности (риска). Чтобы учесть полную вероятность осуществления того или иного энтропийного эффекта, необходимо принять горизонт предвидения как бесконечный. Но при этом степень неопределенности также возрастает до бесконечности. Поэтому в практических расчетах горизонт предвидения следует ограничить рамками, достаточными для проявления всех значимых энтропийных эффектов от внедряемой инновации, на- 
пример, по окончании изучения курсы (дисциплины) (72 часа). Опираясь на исследования, позволяющие произвести расчет энтропийного (Исламутдинов, 2008), определим формулу для оценки и отбора инноваций:

$$
\mathrm{CC} \ni=\sum_{i} \pm \Delta \Pi_{i} \times \bigsqcup_{i} \times \mathrm{M}_{i} \pm \mathrm{B}_{i}
$$

где:

СЭЭ - суммарный энтропийный эффект, в баллах;

$\Delta \Pi_{i}-$ ожидаемое изменение ключевого показателя затрагиваемой инновацией системы под номером $i$, в соответствующих единицах измерения; (если ожидаемое изменение ключевого показателя затрагиваемой инновацией системы уменьшает уровень энтропии, то $\Delta \Pi_{i}$ берется со знаком «минус»; если увеличивает уровень энтропии, то со знаком «плюс»).

$Ц_{i}$ - цена единицы измерения ключевого показателя затрагиваемой инновацией системы под номером $i$, баллов;

$\mathrm{M}_{i}$ - масштаб эффекта в системе, затрагиваемой инновацией, под номером $i$;

$\mathrm{B}_{i}$ - вероятность осуществления эффекта в системе, затрагиваемой инновацией, под номером $i$.

Цена единицы измерения ключевого показателя затрагиваемой инновацией системы определяется по формуле

$$
\bigsqcup_{i}=\frac{100}{\left( \pm \Pi_{K_{i}} \pm \Pi_{\mathrm{T}_{i}}\right)}
$$

где:

$\Pi_{\mathrm{K}_{i}}$ - критическое значение ключевого показателя затрагиваемой инновацией системы под номером i, в соответствующих единицах измерения;

$\Pi_{\mathrm{T}_{i}}$ - текущее значение ключевого показателя затрагиваемой инновацией системы под номером i, в соответствующих единицах измерения. Если критическое значение ключевого показателя затрагиваемой инновацией системы (в 
соответствующих единицах измерения) больше, чем текущее значение, тогда $\Pi_{\mathbb{K}_{i}}$ берется со знаком «плюс», а $\Pi_{\mathrm{T}_{i}}-$ со знаком «минус», и наоборот.

В социальной микрогруппа (учебная группа студентов в вузе) энтропия знаний композиции нескольких взаимно независимых моделей (каждого из студентов в группе) равна сумме их энтропий. Отбор инновационных проектов производится по результатам расчетов. Полученные суммы баллов выстраиваются в ранжированный ряд по мере возрастания суммы баллов. Соответственно, для осуществления (внедрения) отбираются инновации, стоящие первыми в ранжированном ряду.

В современном образовательном процессе подготовки обучающихся необходимо учитывать основные положения, которые определяют системы требований к содержанию, организации и методике предметного обучения. Не смотря на то, что в психолого-педагогической литературе содержатся методы качественного и количественного анализа результатов обучения, важной педагогической задачей представляется разработка новых методик, адекватно позволяющих оценить эффективность применения различных образовательных (инновационных) технологий в педагогической практике $[1,2]$. В условиях интерпретации результатов экспериментальных исследования по выявлению эффективности от внедрения инноваций в систему, представляется возможным оценить масштаб эффективности применения инновации в образовательной среде. При этом проведенные расчеты позволят оценить уровень значимость той или иной инновации на основе анализа её энтропийного эффекта, ожидаемый дидактический эффект от внедрения которой возможно определить с помощью энтропийного подхода.

\section{Список литературы}

1. Абрамова С.В., Бояров Е.Н. Основные принципы развития образовательного пространства «Безопасность жизнедеятельности» // В мире научных открытий. 2010. № 4-2. С. 58-60. 
2. Абрамова С.В., Бояров Е.Н. Оценка сформированности профессиональных компетенций у студентов // Психология обучения. № 3. 2001. С. 68-78.

3. Акимова Т.А. Теория организации. М.: Юнити, 2003. 367 с.

4. Исламутдинов В. Ф. Универсальная методика оценки эффективности инноваций // Менеджмент в России и за рубежом. № 4. 2008. С. 137-140.

5. Лавриненко В.Н., Ратников В.П. Концепции современного естествознания / М.: изд-во «Юнити», 2003 г.

6. Смоленцева Т.Е. и др. Использование энтропии в качестве меры порядка и хаоса внутри системы управления обучением // Вестник Воронежского института ФСИН России. № 2. 2011. С. 109-110.

7. Хакен Г. Синергетика. Иерархия неустоичивостей в самоорганизу ющихся системах и устройствах. М. : Мир, 1985. 411 с.

\section{References}

1. Abramova S.V., Boyarov E.N. Osnovnye principy razvitiya obrazovatel'nogo prostranstva «Bezopasnost' zhiznedeyatel'nosti» [The basic principles of educational space "Life Safety"]. V mire nauchnykh otkrytiy, no. 4-2 (2010): 58-60.

2. Abramova S.V., Boyarov E.N. Ocenka sformirovannosti professional'nykh kompetenciy u studentov [Evaluation of formation of professional competencies in students]. Psikhologiya obucheniya, no. 3 (2001): 68-78.

3. Akimova T.A. Teoriya organizacii [Organization Theory]. Moscow: Yuniti, 2003. $367 \mathrm{p}$.

4. Islamutdinov V. F. Universal'naya metodika ocenki ehffektivnosti innovaciy [A universal method of assessing the effectiveness of innovation]. Menedzhment $v$ Rossii i za rubezhom, no. 4 (2008): 137-140.

5. Lavrinenko V.N., Ratnikov V.P. Koncepcii sovremennogo estestvoznaniya [Concepts of modern science]. Moscow: «Yuniti», 2003. 
6. Smolenceva T.E. i dr. Ispol'zovanie ehntropii v kachestve mery poryadka $i$ khaosa vnutri sistemy upravleniya obucheniem [The use of entropy as a measure of order and chaos within the learning management system]. Vestnik Voronezhskogo instituta FSIN Rossii, no. 2 (2011): 109-110.

7. Khaken G. Sinergetika. Ierarkhiya neustoichivostey $v$ samoorganizu yuschikhsya sistemakh $i$ ustroystvakh [Synergetics. The hierarchy in self neustoichivostey yuschihsya systems and devices], Moscow : Mir, 1985. 411 p.

\section{ДАННЫЕ ОБ АВТОРАХ}

Бояров Евгений Николаевич, доцент кафедры безопасности жизнедеятельности и гражданской обороны, кандидат педагогических наук, доцент

Абрамова Светлана Владимировна, заведующая кафедрой безопасности жизнедеятельности и гражданской обороны, кандидат педагогических наук, доцент

Сахалинский государственный университет

ул. Ленина, 288, г. Южно-Сахалинск, 693008, Россия

e.boyarov@mail.ru

\section{DATA ABOUT THE AUTHORS}

Boyarov Evgeniy Nikolaevich, Associate Professor of Life Safety, Ph.D. in Pedagogical Science, A.P.

Abramova Svetlana Vladimirovna, HoD of Life Safety, Ph.D. in Pedagogical Science, A.P.

Sakhalin State University

288, Lenina street, Yuzhno-Sakhalinsk, 693008, Russia

e.boyarov@mail.ru

\section{Рецензент:}

Савостин Н.М., кандидат педагогических наук 